\title{
Hugh Hellmut Iltis
}

\section{$1925-2016$}

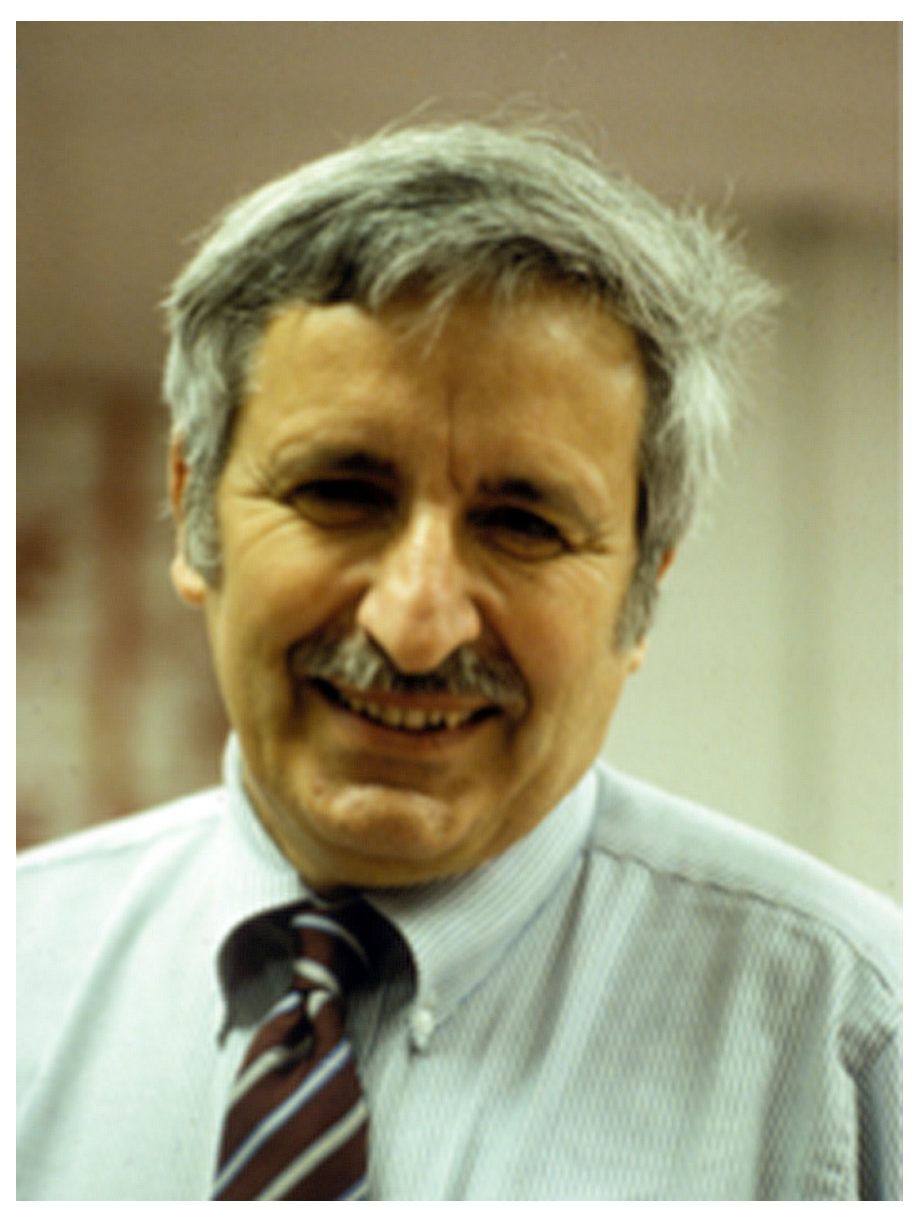

Hugh Iltis en el herbario WIS del Departamento de Botánica de la Universidad de Wisconsin-Madison (fotografía: UW Department of Botany archives, ca. 1990).

\section{J. Antonio Vázquez-Garcíal (D)}

Aguerrido botánico, defensor de la naturaleza y pionero de la biofilia; Profesor Emérito de Botánica y Director Emérito del Herbario WIS en la Universidad de Wisconsin-Madison. Acreedor de numerosos reconocimientos y premios: Contribución Distinguida al Establecimiento de la Reserva de la Biosfera Sierra de Manantlán otorgada por el entonces Presidente de México Miguel De La Madrid Hurtado (1987); Galardón Ambiental Sol Feinstone otorgado por la Universidad del Estado de Nueva York-
Syracuse (1990); Condecoración Nacional al Mérito en Conservación, por la Federación Nacional de Vida Silvestre (1992); Premio al Servicio de la Sociedad de Biología de la Conservación, la mayor en su tipo (1994); Galardón Asa Gray por la Sociedad Americana de Taxónomos de Plantas, máximo reconocimiento en el campo de la Taxonomía (1994); Medalla Luz María Villareal Puga de la Universidad de Guadalajara (1994); Premio al Mérito Botánico por la Sociedad Americana de Botánica (1996);

1 Universidad de Guadalajara, Instituto de Botánica, Departamento de Botánica y Zoología. jvazquez@cucba.udg.mx DOI: http://dx.doi.org/10.21829/abm119.2017.1226

Citar como: Vázquez-García, J. A. 2017. Obituario Hugh Hellmut Iltis. Acta Botanica Mexicana 119: 7-13. DOI: http://dx.doi.org/10.21829/abml19.2017.1226 
Doctorado Honoris causa otorgado por la Universidad de Guadalajara (2006). Además, se han dedicado en su honor 29 taxones, entre ellos un género y 25 especies de plantas de diversas familias. Próximamente (22 de abril de 2017) será instalado en el Salón de la Fama de Conservación en Wisconsin (2016-2017).

Hugh Iltis nació en Brno, Checoslovaquia el 7 de abril de 1925 y falleció a la edad de 91 años el 19 de diciembre de 2016 en Madison, Wisconsin, E.U.A. Fue precedido en la muerte por su padre Hugo, su madre Anni, su hermano Wilfred, su primera esposa, Grace Schaffel, y su tercera esposa, Sharyn Wisniewski. Sobreviven su segunda esposa, Carolyn Merchant y sus cuatro hijos, Frank y Michael de Madison, David de Salt Lake City, y John de Minneapolis.

Su padre, Hugo Iltis, fue un botánico, genetista, naturalista y educador judío-alemán y biógrafo de Gregorio Mendel, el fundador de la genética. Además, por su activismo político de izquierda, fue blanco de los nazis y con la ayuda de Albert Einstein, con quien mantenía correspondencia, la familia abandonó Brno trasladándose a los Estados Unidos de América en 1938 y estableciéndose en Virginia. Cuenta su hijo Michael que Hugh compartió la amenaza nazi de su padre cuando su familia apenas logró escapar de Checoslovaquia tres semanas antes de la invasión nazi en 1939, ya que para llegar a la costa, con el fin de abordar un barco a Inglaterra, tuvo que tomar un tren de medianoche a través de Stuttgart, y que cuando la Gestapo (policía secreta oficial nazi) inspeccionaba el tren sacaron a 10 personas, mientras la madre de Hugh fingía ser la esposa de un diplomático francés y el joven Hugh fingía estar dormido. A los 14 años tenía su propia colección de especímenes de herbario (Fig. 1A), la cual tuvo que vender a la Universidad de Tennessee para ayudarse en sus estudios. Tras iniciar su licenciatura en la Universidad de Tennessee, Hugh H. Iltis ingresó al Ejército de los Estados Unidos durante la Segunda Guerra Mundial (Fig. 1B), pasando en Europa de 1944 a 1946 como médico, interrogador de oficiales alemanes capturados y más tarde como oficial de inteligencia, preparando documentos para los juicios de Núremberg.

\section{ACADEMIA}

Hugh aprendió botánica y genética de su padre y él mismo fue desarrollando un interés genuino por la taxonomía y la evolución. Obtuvo su licenciatura en la Universidad de Tennessee y bajo la asesoría de Aaron J. Sharp (gran amigo y coautor del mexicano-español Faustino Miranda), el joven Hugh desbordó su interés en biogeografía y en floras del Terciario de Norteamérica, así como en florística de Norteamérica. Además, los horribles relatos de Sharp sobre la explosión demográfica en México y la destrucción del bosque en México le impresionaron fuertemente sobre las consecuencias ecológicas negativas del incremento peligroso de la población humana. Obtuvo su doctorado en la Universidad de Washington y el Jardín Botánico de San Luis, Missouri, estudiando con Edgar Anderson. Robert Woodson condujo a Hugh en el estudio de la familia Capparidaceae, mientras que en apoyo a Julian Steyermark, a cargo del proyecto Flora de Missouri, Hugh recolectó especímenes de los Ozarks y adquirió mayor conciencia sobre la importancia de la conservación. Además, siendo estudiante de posgrado viajó al trópico en Costa Rica, donde su apreciación por la naturaleza se convirtió en un gran reto para él de contribuir a conservarla.

Después de tres años de dar clases en la Universidad de Arkansas, Iltis fue profesor de botánica y director del Herbario WIS durante casi 40 años (1955 a 1993), en la Universidad de Wisconsin-Madison, UW-Madison (Fig. 1C). Enseñando sus cursos con entusiasmo y talento dramático, educó a los estudiantes sobre la importancia de integrar taxonomía, biogeografía, ecología, evolución y conservación. Gracias a su intenso activismo el herbario WIS logró alcanzar más de un millón de especímenes de plantas vasculares, incluyendo más de 15,000 especímenes de la Sierra de Manantlán, México, la mayoría identificados por especialistas.

Buscando impulsar la botánica en los trópicos, el vestíbulo de Birge Hall, del Departamento de Botánica de la UW-Madison, durante unos cuantos años fue ocupado (para consternación de algunos administradores) con libros y revistas científicas para donar, llenando en última instancia dos semirremolques con destino a la Univer- 

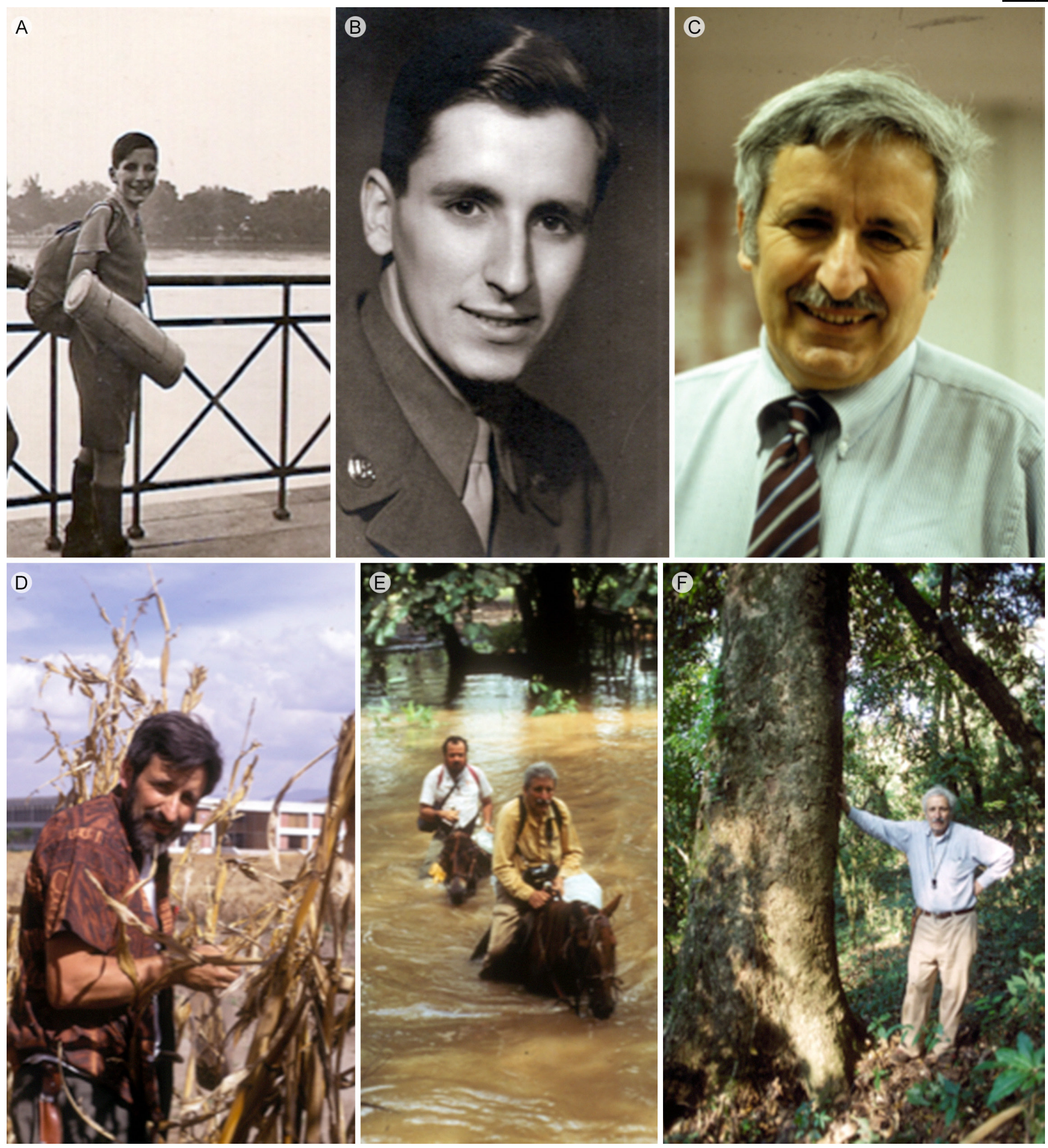

Fig. 1. A. High H. Iltis (HHI) con su primer vasculum para recolecta de plantas en el río Danubio, Checoslovaquia (fotografía por: Iltis family archives, ca. 1937); B. HHI en el ejército de Estados Unidos durante la 2da Guerra Mundial, como médico e interrogador de oficiales alemanes prisioneros de guerra (fotografía por: U.S. Army, 1944); C. HHI en el herbario WIS del Departamento de Botánica de la Universidad de WisconsinMadison (Fotografía: UW Department of Botany archives, ca. 1990); D. HHI en las parcelas de"teocintle" del Dr. George Beadle en Centro Internacional para el Mejoramiento de Maíz y Trigo, Chapingo, México (fotografía por: Theodore S. Cochrane, 30 de noviembre de 1971); E. HHI con el Dr. Alfredo Grijalva, en "cacería" de "teocintle" en Apacunca, Nicaragua ¡Los caballos comenzaron a nadar! (fotografía por Bruce Benz, 8 de octubre de 1991); F. HHI con su especie endémica favorita, Magnolia iltisiana, en la ahora Estación Científica Las Joyas, Reserva de la Biosfera Sierra de Manantlan (fotografía por: undertermined participant of the Society for Conservation Biology field trip, 12 de junio de 1994). 
sidad de Guadalajara. En años posteriores, Hugh continuó coordinando donaciones de publicaciones científicas principalmente a las bibliotecas de UW-Madison.

\section{INVESTIGACIÓN BOTÁNICA}

$\mathrm{Su}$ investigación taxonómica se centró en la familia Capparidaceae y en el género Zea que incluye el maíz y los teocintles (Fig. 1D), trabajando principalmente en México y en el Neotrópico.

\section{Origen y evolución del maíz}

Uno de sus ex alumnos, John Doebley, sostiene que el Dr. Iltis ha sido uno de los principales contribuyentes al estudio de la evolución del maíz durante un cuarto de siglo. Su estudio sobre el origen del maíz, su co-descubrimiento con Rafael Guzmán (de la Universidad de Guadalajara) del teocintle diploide perenne y su teoría de transmutación sexual catastrófica tuvieron gran eco durante décadas. Se señalaron fallas y se propusieron nuevas consideraciones a las postulaciones teóricas de Paul Mangelsdorf y George Beadle. Las aportaciones del Dr. Iltis en este campo de la ciencia se reconocen en Maydica (1990, no. 35), revista internacional sobre investigación en maíz.

\section{La Tercera Expedición de Teocintles (diciembre 1979 - enero 1980)}

Iltis dirigió numerosas expediciones a muchas partes del mundo para buscar nuevas especies de plantas, viajando en mulas o a caballo cuando era necesario (Fig. 1E). Hugh realizó numerosas exploraciones botánicas a la Sierra de Manantlán durante casi siete años en compañía de muchos botánicos de la Universidad de Guadalajara (Rafael Guzmán Mejía, Luz María Villarreal de Puga, Servando Carvajal, Antonio Vázquez, Eugenio Nieto, Francisco Santana, Enrique Jardel, Miguel Cházaro, Ramón Cuevas, Nora M. Núñez, Luis Guzmán y Manuel Rosales), así como con estudiantes y profesores de universidades estadounidenses (Edwards Beals, Bruce Benz, Theodore S. Cochrane, John Doebley, Emmet Judziewics, Robert Kowal, Penny Matekeitas, Robert R. Nault, Michael Nee, Stephen Solheim, Paul Sorensen, Donald Waller, Mark A.
Wetter y Sharyn Wisniewski). Sin embargo, fue la tercera expedición de teocintles a la Sierra de Manantlán la más extraordinaria y prolífica de todas, y una de las más comentadas por él en sus clases, la cual tuvo como objetivo localizar poblaciones adicionales del nuevo teocintle Zea diploperennis (ahora una especie internacionalmente conocida) y caracterizar los ecosistemas de montaña. Fue una de las experiencias botánicas más memorables, compartida entre 19 científicos, diez de ellos de la Universidad de Wisconsin-Madison y cinco de la Universidad de Guadalajara. Todos esperábamos el Año Nuevo y la Década Nueva acampando con una docena de carpas y al calor de una fogata en El Almeal (“Cloud Camp"), a 2000 $\mathrm{m}$ de altitud, con una maravillosa vista cercana de acantilados enormes y niebla. Todo el mundo estaba ansioso por ver la luz del día para iniciar un maravilloso viaje de observaciones y descubrimientos. El Dr. Iltis recogía todas las plantas de floración y fructificación que veía, incluso a altas horas de la noche. Anotó, con su marcador negro, sus números de recolecta en los periódicos, en las estacas y en las frutas secas. Cuando vio por primera vez las flores purpurinas de Canavalia villosa, recuerdo que dijo algo como esto: “ ¡Miren eso!, esas bellas flores colgantes $¿ \mathrm{Se}$ trata de una leguminosa?" El inolvidable viaje remató con el avistamiento de una gran población del recién descubierto teocintle, Zea diploperennis, en Las Joyas.

Iltis es autor de docenas de artículos científicos y capítulos de libros y escritos ambientales, coautor del Atlas de Wisconsin Prairie and Savanna Flora, co-escrito por Theodore Cochrane, coautor de la Flora de Manantlán, obra de colaboración internacional entre la Universidad de Guadalajara y la Universidad de Wisconsin-Madison. Es autor y/o coautor de más de doscientos taxones nuevos para la ciencia, incluyendo la familia Setchellanthaceae y nueve géneros.

El Dr. Iltis dirigió a 45 estudiantes de posgrado, entre ellos: Alvin Yoshinaga, Alwin Gentry (Bignoniaceae), Antonio Vázquez (Magnoliaceae), Bruce Hansen, Emmet Judziewicz (Poaceae), Don Ugent, F. Utech, George Schatz (Annonaceae), Gottlieb Noamesi, K. Roe, Linda 
Leigh, Luz María González Villarreal (Clethraceae), Michael Nee (Solanaceae), Miguel Cházaro (Loranthaceae), Ron Liesner, Shoichi Kawano, Scott Mori (Lecythidaceae), Theodore Cochrane (Cleomaceae) y William Alverson (Bombacaceae). Además de ser mentor moral de muchos otros: Rafael Guzmán (Conservación), Leticia Hernández (Conservación), Aarón Rodríguez (Solanaceae), Sarahi Contreras (Conservación), Oscar Cárdenas (Conservación) y Carlos Palomera (Conservación).

\section{CONSERVACIÓN DE LA BIODIVERSIDAD Y RECURSOS GENÉTICOS}

Los esfuerzos de preservación de Hugh tuvieron éxito, en Wisconsin, Hawái y México. Iltis fue co-fundador en 1960 del Capítulo Wisconsin de la Conservación de la Naturaleza. En 1967 instigó la formulación de la Ley de Áreas Naturales de Hawái, promulgada en 1970.

\section{Pionero de la Biofilia}

Desde 1964, Hugh afirmaba que las razones esenciales por las que debíamos preservar el mundo natural era por nuestra adaptación genética y por nuestra necesidad innata por la belleza natural y la diversidad biológica, tema reiterado en sus charlas y notas ambientales. En la portada de su ejemplar del libro de Biofilia, de autoría de Edward O. Wilson (padre de la sociobiología), figura la inscripción de Wilson: "A HHI, el pionero en el campo". Además, el Dr. Iltis argumentaba que los biólogos sistemáticos y ambientales, por su grado de especialización, cargaban consigo la responsabilidad social de luchar por la preservación. En defensa de las flores, de las mariposas, de las ballenas, de las aves y de los niños, Iltis se promulgó reiterada y enérgicamente contra el consumo estrafalario de las posesiones materiales, la destrucción de la diversidad biológica y el aumento insostenible de la población humana.

\section{Impacto en la Industria de Tomates}

De las 13 especies conocidas de tomates silvestres del mundo, Iltis descubrió dos de ellas. En su viaje a Perú en 1962-1963 con su estudiante doctoral Don Ugent y las es- posas de ambos, con un apoyo de $\$ 21,000$ dólares otorgado por la Fundación Nacional de la Ciencia, describieron Lycopersicon chmielewskii y Lycopersicon parviflorum cuando la primera demostró, 17 años más tarde, que vale millones de dólares al año para la industria de tomates, un rendimiento de más de diez mil veces la inversión en una investigación pequeña, valor que puede ser minimizado por el valor aún desconocido de los más de 8000 especímenes recolectados en ese viaje.

\section{El Hallazgo Zea diploperennis (1979)}

$\mathrm{Su}$ sensacional co-descubrimiento del teocintle perenne con Rafael Guzmán Mejía, de la Universidad de Guadalajara, como resultado de la tarjeta botánica anual de Año Nuevo, que Hugh envío a la profesora Luz María Villarreal de Puga en 1976 con una ilustración de un teocintle perdido, fue publicado en la revista Science (1979), lo cual acaparó los reflectores y la atención de diversas revistas científicas y periódicos, convirtiéndose ya en una Leyenda. Una nota de esta especie fue publicada en la portada del New York Times el lunes 5 de febrero de 1979 en un artículo de Walter Sullivan titulado "Esperanza de crear maíz perenne a partir de un nuevo descubrimiento botánico". Iltis reconoció de inmediato el potencial económico de esta especie, siendo un diploide perenne relacionado al cultivo del maíz. Subsecuentemente, el Dr. Lowell "Skip" Nault demostró en su artículo publicado en la revista Desert Plants (1981) que Zea diploperennis es resistente a varias enfermedades virales del maíz.

\section{Las Magnolias en peligro y el establecimiento de la Estación Científica Las Joyas, Jalisco (1985)}

En 1984, durante una excursión con el botánico mexicano Rafael Guzmán, el Dr. Iltis notó que numerosos árboles viejos, incluyendo majestuosas magnolias de $30 \mathrm{~m}$ de altura, estaban marcadas en su base (Fig. 1F) para ser derribadas. Una notificación oportuna de esta tala ilegal, primero a la Maestra Luz Ma. Villarreal de Puga y luego al Gobernador del Estado, impulsó la primera acción oficial de conservación por parte del Gobierno del Estado: la compra de un área forestal de 1245 ha, en Las Joyas, cerca 
de la cima de la Sierra de Manantlán, que poco después condujo al establecimiento de La Estación Científica Las Joyas para proteger el teocintle Zea diploperennis, sus bosques de pino-encino y sus bosques nubosos (bosques mesófilos de montaña). Como parte de mi grado de Maestría en Botánica, varias nuevas especies de Magnolia fueron descritas para México y América Central (Brittonia, 1994), las poblaciones de magnolia de Las Joyas (Fig. 1F) fueron descritas como una nueva especie, Magnolia iltisiana, para honrar la invaluable trayectoria del Dr. Hugh Iltis en el conocimiento y la preservación de La Sierra de Manantlán.

\section{La Flora de Manantlán y el decreto de estableci- miento de una Reserva de la Biosfera (1987)}

En un esfuerzo conjunto entre la Universidad de Guadalajara y la Universidad de Wisconsin-Madison, en el que participaron Hugh Iltis, Ted Cochrane, Ramón Cuevas y otros coautores, se creó un compromiso duradero (13 años) para producir un inventario anotado de la Flora de Manantlán (ahora con más de 3000 especies), en el occidente de México, una de las obras más autorizadas de este tipo, con la participación de más de 130 taxónomos de plantas de todo el mundo. Las versiones preliminares de este esfuerzo ayudaron a justificar la propuesta que condujo al establecimiento (en 1987) de ca. 140,000 hectáreas de la Sierra de Manantlán como Reserva de la Biosfera, dentro del Programa MAB-UNESCO, un área casi dos tercios del tamaño del parque nacional Great Smokey Mountains. Fue la primera vez que se fundó una reserva de biosfera internacional partiendo de proteger una especie rara y endémica, cuyo germoplasma posee la única fuente conocida de resistencia genética a diversos virus del maíz y a la enfermedad del óxido de maíz; por lo tanto, es potencialmente de enorme valor económico. El Inventario Florístico de la Sierra de Manantlán continuó como un proyecto inicial dentro del acuerdo de cooperación trinacional entre la Universidad de Guelph (Canadá), UW-Madison y UdeG (México). También fue fundamental para el plan maestro de manejo de la Reserva de la Biosfera. Lejos de encontrar más poblaciones de teocintle en los primeros días, hubo recolectas prolíficas de plantas vasculares, algas, hongos, pteridofitas, musgos y aves. Así fue que Hugh Ilits inició el interés por conocer no solo las nuevas poblaciones de teocintles, sino toda la Flora de Manantlán, y fue el primero en señalar la necesidad de proteger todas esas cadenas montañosas de extraordinaria biodiversidad. El Dr. Iltis afirmaba con insistencia: "debes hacer todo lo posible por proteger estas maravillosas montañas".

\section{ACTIVISMO AMBIENTAL}

$\mathrm{Su}$ papel en todos los aspectos de su carrera fue animar a la gente, confrontar a la gente con la dura realidad de lo que debe hacerse para preservar la calidad del medio ambiente natural para la supervivencia humana y para el estudio científico.

\section{Prohibición del DDT en Wisconsin}

En 1968 Hugh formó parte de un pequeño grupo que incluía a su colega Orie Loucks cuyo activismo llevó a la prohibición del DDT en Wisconsin, que a su vez condujo en última instancia a una prohibición nacional. Citando a Lorrie Otto en el periódico Capital Times Madison WI (14 de julio de 2007): "Triunfo altísimo: La prohibición del DDT trajo a las águilas y comenzó en Wisconsin”, "el regreso del águila es algo que todos los estadounidenses deberían celebrar..." Entonces, no existían las normas de especies en peligro de extinción ni las leyes sobre aire limpio y agua limpia.

\section{Control de la Natalidad}

Desde 1974 Hugh Iltis ya se manifestaba como un firme partidario de los derechos al aborto. Se documenta que en tiempos en que era ilegal en Wisconsin mostrar públicamente un anticonceptivo humano, Hugh desafiaba a la policía a arrestarlo, sosteniendo una percha en forma de espiral, decía: "esto es un DIU para una ballena” y que también solía mostrar un costal y decir sería un condón de mamut, mientras que una bolsa de pan francés puede ser un condón de elefante. Hugh relataba la historia de Mechai, quien casi por sí solo redujo la tasa de natalidad de Tailandia en más de cuatro veces al encontrar una línea en la escritura bu- 
dista que decía: "Demasiadas bocas causan sufrimiento", y que pronto se alcanzó la cifra de 20,000 monjes y 200,000 educadores con este mensaje. En el México preponderantemente católico, Hugh hizo una monumental contribución a la conservación de la biodiversidad en el este, centro y oeste del país, a finales de los años '70 y principios de los '80 del siglo XX. Hablaba sobre la urgente necesidad de preservar los ecosistemas en momentos en que ni siquiera los biólogos eran conscientes de la crisis de la biodiversidad que se avecinaba, y exhortaba a la audiencia a usar condones cuando la mayoría de la gente no tenía ni idea de la explosión demográfica en México y sus impactos potenciales. Durante su discurso haría una pausa y diría a la multitud: "Por favor, usen condones ¿Lo harán, verdad?".

El Dr. Iltis preparó cuidadosamente cada uno de sus discursos con el objetivo de transformar la sociedad para un mundo mejor en pro de los niños, las mariposas y las ballenas. Cada diapositiva fue sabiamente seleccionada para transmitir un mensaje científico, cultural o fuertemente emocional. Su gran sensibilidad, ingenio, sabiduría, experiencia, así como su gesticulación y potente voz (clara y firme) hicieron de él un extraordinario elocuente y persuasivo orador.

Hugh fue un pensador sobresaliente y un guerrero, que en el día a día declaraba la guerra a la "ilimitada ignorancia y estupidez humana", en favor del medio ambiente y del mundo natural. Quienes le conocimos de cerca aprendimos de él mucho más de lo que ahora podemos reconocer, y ahora esperamos que su visión y legado cultural continúe y se transmita a las generaciones presentes y futuras, así como su pasión por la naturaleza y las semblanzas de su admirable vida. 
